\title{
Mobile Data Gathering using Mobile-MIMO Scheme in Wireless Sensor Networks
}

\author{
Madhu Priya ${ }^{1}$ and Himani Verma ${ }^{2}$ \\ Student, Gautam Buddha University, Greater Noida, India ${ }^{1,2}$
}

\begin{abstract}
In this paper, we have proposed a three-layer framework for mobile data gathering in wireless sensor networks, which includes the sensor layer, cluster head, \& mobile collector layer. Distributed load balanced clustering and dual data uploading is employed in our framework. Good scalability, long network lifetime and low data collection latency are the main objectives to be achieved. A distributed load balance clustering algorithm is proposed at the sensor layer in which the sensor self-organizes themselves into clusters. In our scheme multiple cluster heads are generated in each cluster to balance the work load and preside dual data uploading. The multiple cluster heads cooperate with a cluster to perform energy saving inter-cluster communications. At the mobile layer two cluster heads are enabled simultaneously to upload data to sencar by utilizing mobile MIMO (multiple-input and multiple-output) technique. By visiting selected and associated pairs polling points, sencar can efficiently gather data from cluster heads. To evaluate the effectiveness of proposed mobile-MIMO scheme extensive simulations are conducted.
\end{abstract}

Index Terms: Wireless sensor networks (WSNs), data collection, load balancing, mobile-MIMO.

\section{INTRODUCTION}

Wireless communications has capable the development of low cost, low power, multifunctional sensor nodes that are compact in size and can communicate over short distances unrestrainedly[3]. Sensor nodes consist of sensing, data processing and communicating components. A sensor network is composed of a wide range of sensor nodes which are generally densely deployed and scattered randomly over a sensing field and are left uninstructed after being deployed, which makes it burdensome to recharge or replace their batteries much fasters that other sensors due to more traffic. The network connectivity and coverage may not be guaranteed if the sensor around data sink depletes their energy. Due to these persuasions, it is essential to design an energy-efficient data collection scheme that consumes energy uniformly so that a long network lifetime can be achieved [1].

Wireless sensor networks are an integral part of military command, control communications, computing, intelligence, surveillance, targeting system etc and also for monitoring the machine health. They are also deployed in critical applications like gas monitoring in mines, fires detection and for reporting the vital information to the end users [4].

As sensing data in some applications are time sensitive so the data collection has to be performed in a specified time frame. Therefore, an efficient data collection scheme should aim at good scalability, long network lifetime and low data latency.

Different approaches have been proposed for efficient and enhanced data collection in the literature see for example [4]-[24]. Focusing on these works, we can classify them into three broad categories. The first category is the enhanced relay routing in which data is relayed among sensors apart from that a load balance, schedule pattern and data redundancy are also taken in consideration. The second category is clustering in which the sensors organize into clusters and each cluster has a leader known as cluster head. A cluster head may be elected or pre-assigned by the members of the cluster and further the cluster head collects the data within the cluster and transfer to the destination i.e. data sink. The clustering technique is widely perused for increasing the lifetime as well as scalability of the networks and is effective in local data aggregation as it can reduce the collisions and balance load among the sensors [10].

The third category is to make use of mobile collectors which takes the burden of data routing from sensors in a network. These works have provided efficient solutions to data collection schemes in WSNs but their effectiveness have been noticed. In relay routing technique prolonging network lifetime does not necessarily takes place by minimizing energy consumption on the forwarding path of the network. In cluster head based schemes, the cluster head will inexorably consume the more energy compared to the sensors due to handling data traffic i.e. intra cluster aggregation and inter cluster data forwarding. In mobile collectors schemes the non-uniform energy consumption ameliorate which may result in inadequate data collection latency.

Based on this observance, in this paper, we propose a three layer frame work for mobile data collection which is named as load balanced clustering and dual data uploading. The main incentive is to exploit distributed clustering for scalability, to hire mobility for energy expenditure and uniform energy consumption and to take advantage of multiuser multiple-input and multiple-output (MIMO) technique for concurrent data uploading to shorten data latency. 
Vol. 6, Issue 4, April 2017

The main objectives and contributions of these works can be summarized as follows. Firstly, a distributed algorithm is forth put which organize sensors into clusters, where each clusters have multiple cluster heads. In contrast to the previous clustering techniques, our algorithm manages and balances the load of intra cluster aggregation, inters aggregation and empowers dual data uploading between multiple cluster heads and the mobile collector. Secondly, to perform an energy-efficient inter cluster transmissions the multiple cluster heads within a cluster can cooperate treasonably with each other. Distinct from other hierarchical schemes [17], in our algorithm, the cluster heads do not depend on the data packets from other clusters which essentially ameliorate the burden of each cluster head. Alternative, the forwarding paths among clusters are only used to route small-sized identification (ID) information of cluster heads to mobile collector for enhancing and improving the data collection tour. Thirdly, a mobile collector is installed with two antennas (called sencar) to allow simultaneous uploading from two cluster heads by using MUMIMO communication. The sencar visit each cluster and collects the data from the cluster heads. The stop locations inside each cluster is elected by the sencar and the sequence of visit is also determined by them such that data collection can be done in minimal time our work mainly accomplishes from the other mobile collection schemes [20] in the utilization of MIMO technique which shorten data transmission latency by enabling dual data uploading which eventually leads to a data collection tour with short moving trajectory and short data uploading time.

\section{LITERATURE REVIEW OF PREVIOUS WORKS}

\subsection{RELAY ROUTING AND CLUSTERING SCHEMES:}

Relay routing is the most simplest and effective approach to routing messages to the data sink in a multi-hop fashion. Cheng, et al [4] to avoid congestions a coordinated transfer schedule was devised by choosing alternate routes. Wu, et al [8] studied data gathering process which aimed to conserve energy and extend network lifetime. A tree based topology is used for collecting data from sensor nodes. Construction of a data gathering tree is studied when a single base station is present in the network. An algorithm was designed that starts from an arbitrary tree and iteratively reduces the load on the bottleneck nodes (the nodes likely to deplete energy due to high degree or low remaining energy). Xu, et al [5] studied the relay nodes deployments to extend the lifetime of the network. Appropriate node placement is an effective approach for optimizing the desired goals. By placing the relay nodes strategically the network efficiently can be improved. Gnewali, et al [6] studied collection tree protocol (CTP). The two principles for wireless routing protocols- data path validation and adaptive beaconing were evaluated 12 different test beds ranging in size, comprising seven platforms and six different link layers used for studying CTP. CTP is a routing protocol which computes routes to a single or a number of designated sinks in a wireless sensor networks. The mechanisms allow CTP to remain efficient, robust and reliable even in dynamic topology. CTP offers 90-99\% packet delivery in dynamic environments and is robust to topology changes and failures. However, some nodes lying on critical path may deplete energy as a result of which data collection performance will be deteriorated. Another approach is to allow nodes to form cluster and cluster heads for reducing the number of relays [10]. Heinzelman, et al [10] a cluster formation schemes was proposed called as a "LEACH" in which smallest expected number of clusters were formed LEACH enables a new distributed cluster formation technique which includes the self organization of large numbers of nodes and algorithms for adapting clusters. The drawback of this technique was it does not guarantee a good cluster head distribution and also makes an assumption of uniform energy consumption for cluster heads. Younis, et al [11] proposed "HEED" which is a new energy efficient approach for clustering nodes in wireless sensor network. HEED in which metric considered for cluster head selection uses combination of residual energy and cost. Well distributed cluster heads and compact clusters are produced by HEED. Gong, et al [12] based on link quality energy efficient clustering in lossy wireless networks considered. Amis, et al [13] proposed d-hop clustering in which each node is at most d-hops away from a cluster head. A cluster head can also be termed as a scheduler or controller for in-network processing in a cluster based schemes. Zhang, et al [15] to alleviate the collisions among different transmissions efficient scheduling of cluster heads considered. Gedik et al [17] and liu, et al [18] the correlation of sensing data is examined and sensor nodes are dynamically partitioned into clusters. The spatiotemporal correlation is utilized by the cluster heads to minimize the readings for energy saving. MU-MIMO scheme may not be compatible with the traditional single-head clustering schemes. Thus, load balanced multi-head clustering algorithm is proposed in this paper.

\subsection{MOBILE DATA COLLECTIONS:}

The balancing of energy consumptions in the network and connecting the disconnected regions benefits are enjoyed by introducing the mobility for data collection. Shah, et al [19] mobility under random walk is investigated where data is picked up from the neighbouring sensors and buffers by the mobile collector and finally the data is offloaded to the wired access point. However, latency bounds cannot be guaranteed in random trajectory which is required in various applications. Jea, et al [20] data mules traversing the sensing field along parallel straight lines was proposed to control and through multi-hop transmissions the data was collected from the nearby sensors. This proposed scheme works in a 
uniformly distributed sensor network. An efficient moving path planning algorithm was proposed by Ma and Yang [16] to achieve more flexible data gathering tour for mobile collectors. Some turning points on straight lines are determined which is adaptive to sensor distribution and can avoid the obstacles on path effectively. Furthermore, Somasundara et al [25] proposed an algorithm to study mobile elements scheduling in such a way that no data transmission time for mobile data collection can be efficiently reduced by MU-MIMO.

\subsection{MU-MIMO IN WSNs:}

In wireless sensor networks the feasibility of employing MIMO technique is discussed in [27]. It is difficult to mount multiple antennas on a single sensor node thus MIMO scheme is used in WSNs which cooperates multiple nodes to achieve diversity and bit error rate is reduced. The energy consumption in MIMO is higher than traditional SISO (single-input single-output) approach which is another challenge. In [27] it was explained that when the transmission range is larger than SISO. In our scheme two antennas can be deployed on mobile collector thus enable MU-MIMO uploading which reduces data collection latency.

\section{SYSTEM OVERVIEW}

Consist of three layers: sensor layer, cluster head layer and sencar layer. The sensor layer is primary and bottom layer. There are no assumptions on sensor distribution or node capability. It is assumed that each sensor has a capability to communicate only with its neighbours i.e. nodes lying in its transmission range. Initially the sensors are self-organized into clusters and further decide to be either a cluster member or cluster head in a definite manner. The sensors having higher residual energy would become cluster heads in the network. Each cluster has at most M cluster heads where M is a parameter used in the system. The multiple cluster heads formed in the cluster are known as cluster head group (CHG) where each cluster head is peer of others. The algorithm is constructed in such a way that each sensor in a cluster is at least 1-hop away from at least one cluster head. Due to this organization intra-cluster aggregation is limited to single hop. During data aggregation the CHG uses time-division multiple access (TDMA) based technique to avoid collisions and to coordinates the communication among the sensors. After the cluster heads are decided the nodes synchronize their clocks. On arrival of sencar each CHG uploads stored data via. MU-MIMO communications and the local clocks are synchronized with the global clock on sencar using an acknowledgement messages. Finally to avoid the draining energy from the cluster heads reclustering is done periodically in which the cluster heads are rotated among the sensors with high residual energy.

All cluster heads are present in the cluster head layer. The CHG information of each cluster is send to sencar using only inter-cluster forwarding which consists of an identification list of multiple cluster heads in a CHG. Before the sencar departs for the data collection tour the information must be send before. After the information is received the sencar takes the advantage to determine where to stop within each cluster to collect data determine where to stop within each cluster to collect data from its CHG. The connectivity for inter-cluster communication can be guaranteed when duplicated information is send out by the cluster heads in a CHG to achieve spatial diversity for providing reliable transmissions and energy saving [27]. To ensure a degree of connectivity among clusters, the cluster heads can adjust their output power for a desirable transmission range. The top-most layer is the sencar layer which manages the mobility of sencar. There are two major issues at sencar layer. Firstly, we need to determine the locations where sencar stop to communicate with the cluster heads when it arrives at a cluster. Single hop transmissions are used by sencar to communicate with the cluster heads. The sencar is equipped with two antennas while sensor with a single antenna. The data uploading traffic pattern in cluster is many-to-one, where data from different cluster heads converge to sencar. As sencar is equipped with two receiving antennas it can make dual data uploading whenever possible and two cluster heads can upload the data simultaneously. A finite set of locations are considered. The channel state information is measured by sencar before each data collection tour selects the candidate location for data collection. These possible locations are called polling points where sencar stop to perform concurrent data collections. All the polling points are most visited by sencar. Instead, only the selected polling points which are accessible are used by the sencar.

The purpose of this work is to achieve great energy saving and shortened data collection latency. Using the technique called MU-MIMO can greatly increase the data collection time and overall latency can be reduced.

\section{DLBC ALGORITHM}

The distributed load balanced clustering at the sensor layer is presented. The selection of cluster heads is the most vital operation of clustering. The selected cluster heads are expected to have higher residual energy in order to have prolonged network lifetime. The percentage of residual energy of each sensor is used as the initial clustering priority. A set of sensor is assumed, denoted $\mathrm{S}=\{\mathrm{S} 1, \mathrm{~S} 2, \ldots \ldots . \mathrm{SN}\}$, are homogenous and each sensor makes the decision independently on its status based on the local information available. After running the algorithm each cluster will have at most $\mathrm{M}(\geq 1)$ cluster heads and in a cluster each sensor is covered by at least one cluster head. 


\subsection{INITIALIZATION:}

STEP1: Each sensor communicates with all neighbours in its coverage area

$\mathrm{N} \longleftarrow$ (U/U lies in transmission range, $\mathrm{U}$ 回 $\mathrm{S}$ );

STEP2: If sensor node is isolated node, it requests itself to be cluster head.

$\mathrm{N}=\Phi$ then

Set cluster head to id;

Set status to cluster head;

STEP3: Otherwise set status as "tentative" and the percentage of residual energy is given as initial priority.

Init prio Eres/Etot;

STEP4: When cluster head

Set status as tentative;

And cluster head peers

0

$\{\mathrm{U} / \mathrm{L}$ peers $(\mathrm{N})\}$;

STEP5: Prio it prio $+\sum$ cluster head peers init prio;

STEP6: Potential cluster heads and cluster members set to $\Phi$ then Iter $\quad 0$;

\subsection{STATUS CLAIM:}

In this phase each sensor determines its status by updating local information and restraining to be a cluster head. The priority is partitioned into three zones by two thresholds i.e. Th and $\mathrm{Tm}(\mathrm{Th}>\mathrm{Tm})$ which endow a sensor to declare itself as cluster head or member respectively before maximum number of iterations are reached. If sensor priority is greater than Th or less than Tm compared with its neighbours it decides its final status and quit iteration. If a sensor has reached maximum number of iterations and has highest priority over others then it claims to be a cluster head and this process is called as self-driven status transition. The packet is broadcasted by the sensor including ID list to its current candidate peers to be cluster heads, referred to as peer- driven status transmission. If sensor priority is less than or equal to $\mathrm{Tm}$ then it fails to qualify to be a cluster head and quits iteration. It claims to be a cluster member.

\subsection{FORMATION OF CLUSTER:}

In this phase of cluster forming the cluster head decides which sensor should be associated with it. A sensor with tentative status or being cluster member would affiliate itself randomly with a cluster head among its candidate peers for load balancing purpose. Re-clustering is needed when a cluster head is running out of battery energy. The reclustering is performed by sending out re-clustering message to all cluster members. On receiving the message the cluster members switch to the initial phase and a new round of clustering is performed.

\subsection{SYNCHRONIZATION OF CLUSTER HEADS:}

In this final phase local clocks are synchronized among cluster heads in a CHG by a beacon messages. A beacon message with its initial priority and local clock information of each cluster head is sending out to other nodes in CHG. Then the received beacon messages are examined to check if priority of beacon message is higher. The local clock is adjusted according to time stamp of beacon message if priority is higher.

\section{PERFORMANCE EVALUATIONS}

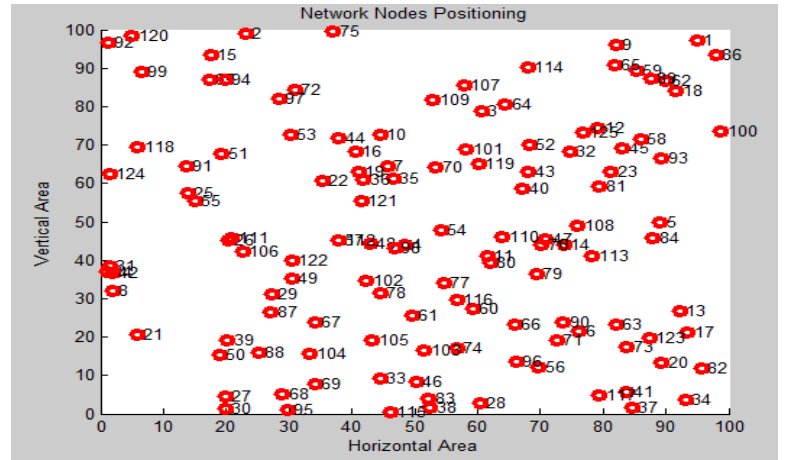

Figure1 random arrangement of nodes

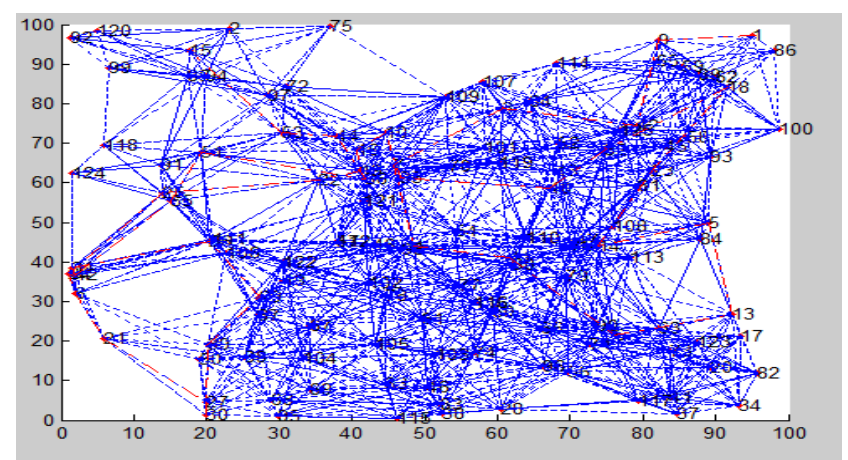

Figure2 network configuration

In this section the performance of our framework is evaluated and compared with other schemes. The figure 1 shows random arrangement of 125 sensors on a $100 * 100$ area. The links between the neighbouring nodes identify the connectivity among the sensors. Figure 2 represents the network configuration with 125 sensors which are distributed over a $100 * 100$ area. 


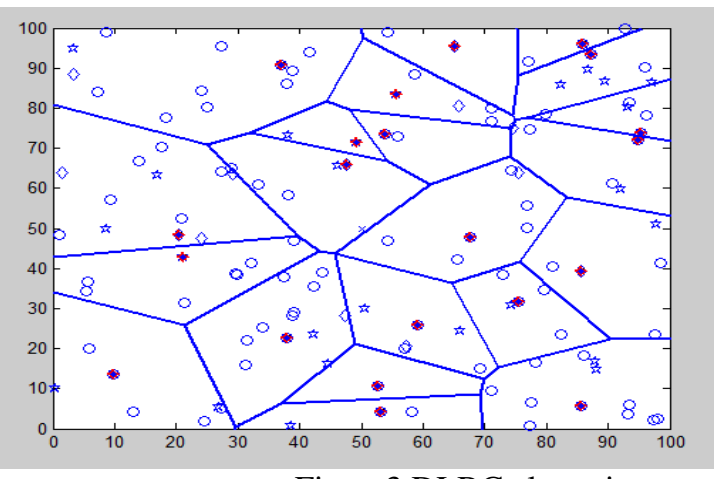

Figure3 DLBC clustering

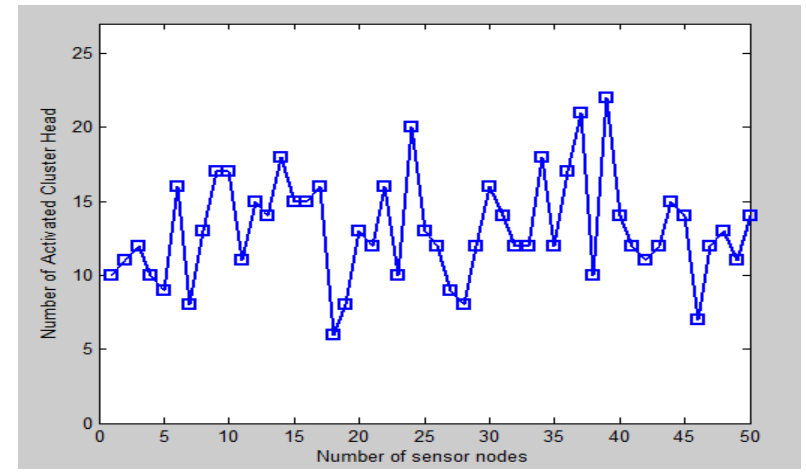

Figure4 initial priority of sensors

Figure3 displays the result of DLBC i.e. distributed load balanced clustering when $\mathrm{M}$ is set as 2 . The sensors are selforganized into clusters where each cluster has two cluster heads. Figure4 represents the number of activated cluster head which are highlighted in blue. It is examined that the final cluster heads have the highest initial priorities in its proximity which is used to validate the requirement that sensors with higher residual energy are selected to be cluster heads. Figure 5 and 6 represent the intra clustered and inter clustered trajectory.

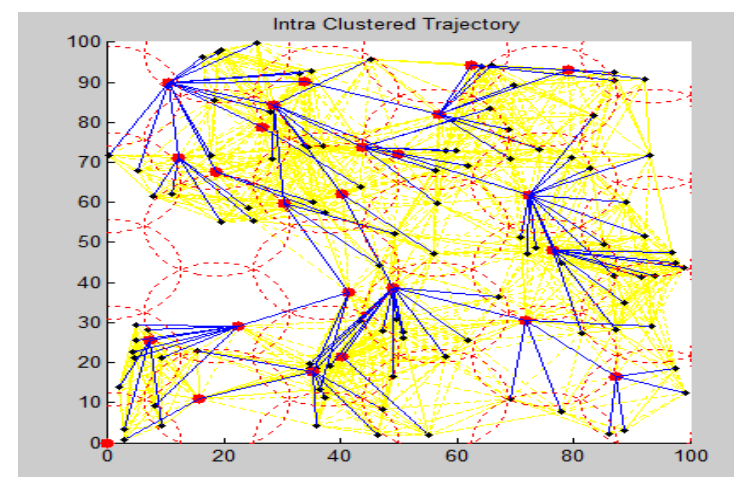

Figure5 Intra clustered

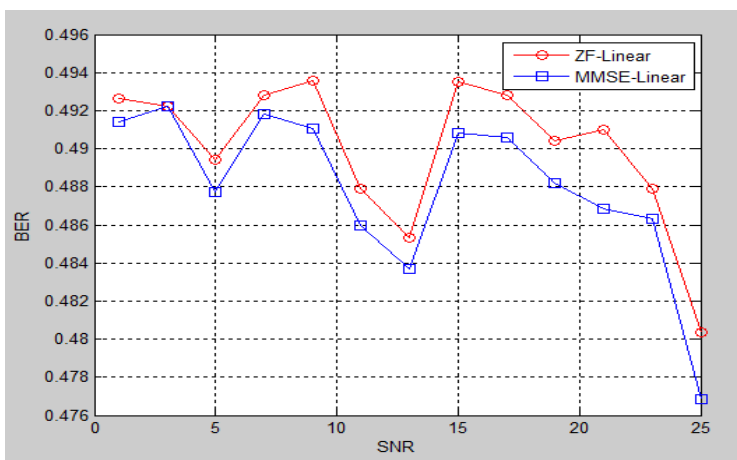

Figure7 ZF \& MMSE

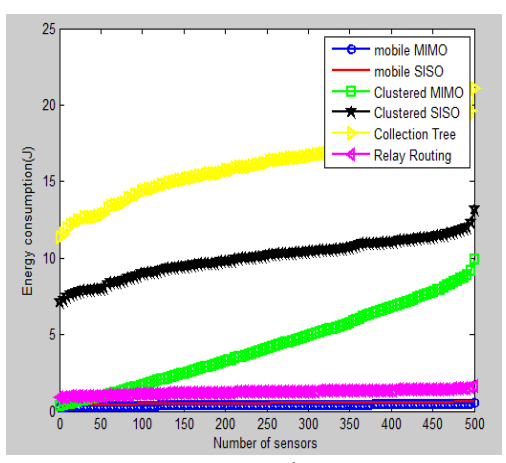

Figure9

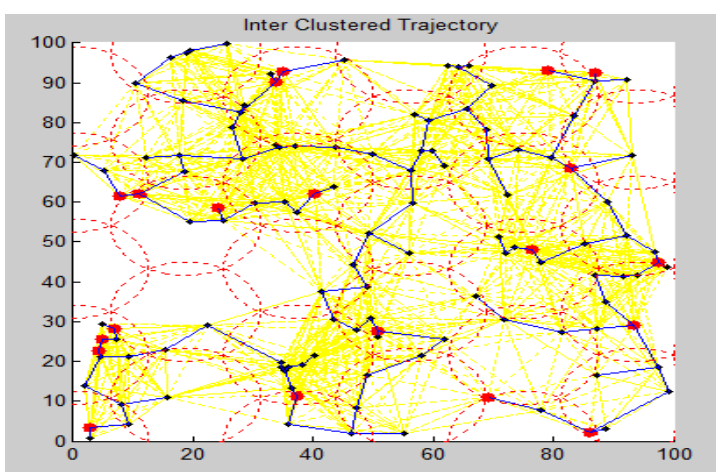

Figure6 inter clustered

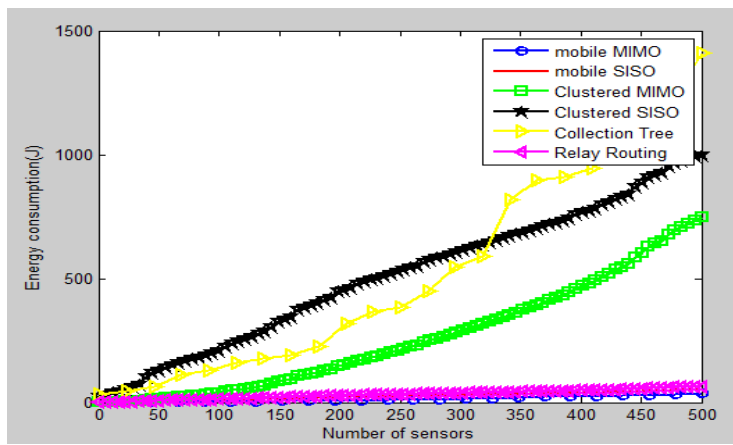

Figure8

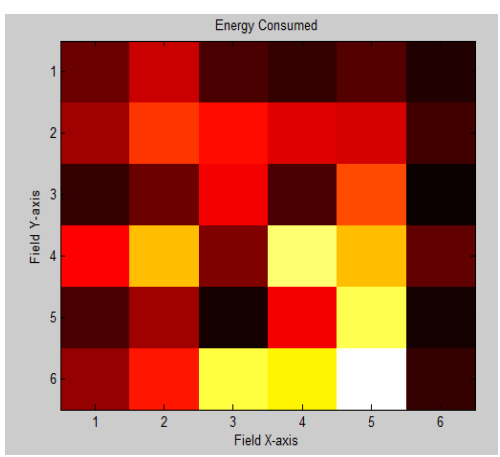

Figure10 
All the transmissions from cluster member to cluster head are included in intra cluster communication. TDMA scheme is considered for the intra cluster period. As we limit all the communications for a cycle in a consecutive period the cluster head does not need to switch between intra and inter cluster communications. The whole duration is classified into multiple identical time slots of length ' 1 ' which is equal to the time required for packet transmission. The cluster members sent packets in these time slots directly to the cluster head. The ' $t$ ' time slots are assigned to each node for the necessary control packets. If a cluster has ' $\mathrm{m}$ ' modes then duration of intra cluster period is given as $\mathrm{m} * \mathrm{t} * 1$.

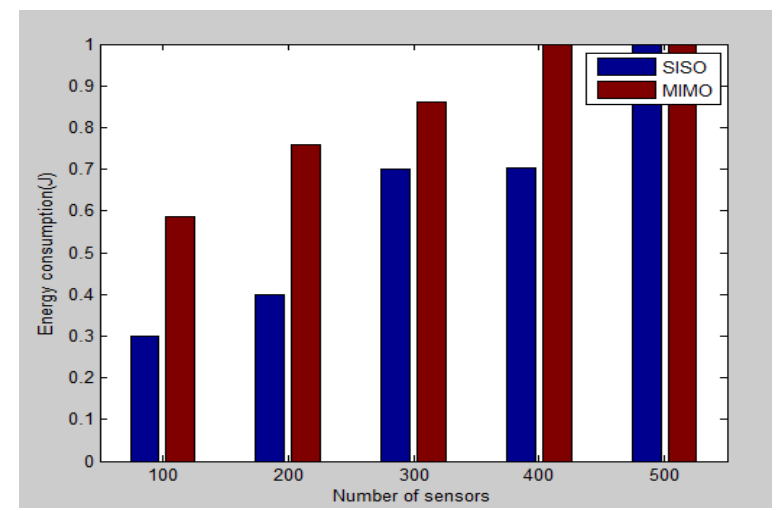

Figure11

The transmissions in the relay network are included in the inter-cluster communication which consists of CHs and the sink. An interval is assigned to each $\mathrm{CH}$ to send all the packets within a given cycle which includes the packets received by it and packets collected from other CHs. Figure7 presents the analysis of the zero forcing (ZF) and minimum mean squared error (MMSE) which is applied to the wireless multi-input multi-output (MIMO) system. Figure8 and figure9 shows the comparison with other schemes. An assumption is made that all the schemes are implemented under same duty cycling MAC strategy. Relay routing is the first scheme to relay messages to static data sink in multi hops. Load balance among nodes can be provided by relay routing along the routing path. Collection tree protocol is the second schemes used for comparison [6]. The third scheme is clustered SISO. In this scheme the sensors form clusters with a single cluster head. In SISO communications, the cluster heads receive data messages and static data sink is relayed in multi-hops. This scheme is further extended to cluster MIMO which allows MIMO communications. The next scheme is mobile SISO for mobile data gathering where in each cluster the sencar stops for data collection and data is uploaded to sink after all clusters have been visited. The last schemes is our proposed scheme i.e. mobile MIMO in which the multiple cluster heads can enable MU-MIMO uploading to sencar in each cluster. Figure8 presents the maximum energy consumption in the network where as figure9 compares average energy consumption per node. In both figure we observe that our proposed mobile MIMO scheme results in least maximum energy consumption. The heat map of energy consumption is shown in figure10. The proposed mobile MIMO method results in less energy consumption. In figure 11 the energy overhead is plotted. The extra energy consumed in MIMO circuitry is quite small which is represented by the gap between mobile SISO and MIMO. The energy consumption in MIMO circuitry is negligible.

\section{CONCLUSIONS}

In this paper, a three layer framework has been proposed for mobile data collection in a WSN. It consists of sensor layer, cluster head and sencar layer and distributed load balanced clustering is employed for sensor self organization and for energy efficient transmission. The result shows that mobile MIMO can reduce energy consumptions greatly. It achieves less data collection time compared to mobile SISO data gathering and energy saving on cluster heads is more. The energy overheads also have been justified and results with different numbers of cluster heads explored in our proposed framework.

\section{REFERENCES}

[1] B. Krishnamachari, Networking Wireless Sensors, Cambridge University Press, Dec. 2005

[2] R. Shorey, A. Ananda, M.C. Chan and W.T. Ooi, Mobile, Wireless, Sensor networks, IEEE Press, Mar. 2006.

[3] I. F. Akyildiz, W. Su, Y. Sankarasubramaniam and E. Cayirci, "A survey on sensor networks," IEEE Commun. Magazine, pp. 102-114, Aug. 2002.

[4] W. C. Cheng, C. Chou, L. Golubchik, S. Khuller and Y.C.Wan, "A coordinated data collection approach: design, evaluation, and comparison," IEEE Jour. of Sele. Areas in Comm., vol. 22, no. 10, Dec. 2004.

[5] K. Xu, H. Hassanein, G. Takahara and Q. Wang, "Relay node deployment strategies in heterogeneous wireless sensor networks",IEEE Trans. on Mobile Computing, vol. 9, no. 2, 2010.

[6] O. Gnawali, R. Fonseca, K. Jamieson, D. Moss and P. Levis, "Collection tree protocol,” ACM Sensys, 2009. 
[7] E. Lee, S. Park, F. Yu, and S.-H. Kim, "Data gathering mechanism with local sink in geographic routing for wireless sensor networks," IEEE Trans. Consum. Electron., vol. 56, no. 3, pp. 1433 -1441, Aug. 2010.

[8] Y. Wu, Z. Mao, S. Fahmy and N. Shroff, "Constructing maximum-lifetime data-gathering forests in sensor networks," IEEE/ACM Trans. Netw., vol. 18, no. 5, pp. $1571-1584$, Oct. 2010.

[9] X. Tang and J. Xu, “Adaptive data collection strategies for lifetime-constrained wireless sensor networks,” IEEE Trans. Parallel and Distributed Systems, vol. 19, no. 6, 2008.

[10] W.R. Heinzelman, A. Chandrakasan and H. Balakrishnan, “An application specific protocol architecture for wireless microsensor networks," IEEE Trans. Wireless Comm., vol. 1, no. 4, Oct. 2002.

[11] O. Younis and S. Fahmy, "Distributed clustering in ad-hoc sensor networks: a hybrid, energy-efficient approach," IEEE INFOCOM, Hong Kong, 2004.

[12] D. Gong, Y. Yang and Z. Pan, "Energy-efficient clustering in lossy wireless sensor networks," Journal of Parallel and Distributed Computing, vol. 73, no. 9, pp. 1323-1336, Sept. 2013.

[13] A. Amis, R. Prakash, D. Huynh and T. Vuong, "Max-min d-cluster formation in wireless ad hoc networks," IEEE INFOCOM, March 2000.

[14] A. Manjeshwar and D.P. Agrawal, "Teen: a routing protocol for enhanced efficiency in wireless sensor networks," IEEE IPDPS, April 2001.

[15] Z. Zhang, M. Ma and Y. Yang, "Energy efficient multi-hop polling in clusters of two-layered heterogeneous sensor networks," IEEE Trans. Computers, vol. 57. no. 2, pp. 231-245, Feb. 2008

[16] M. Ma and Y. Yang, "SenCar: an energy-efficient data gathering mechanism for large-scale multihop sensor networks," IEEE Trans. Parallel and Distributed Systems, vol. 18, no. 10, Oct. 2007.

[17] B. Gedik, L. Liu and P.S. Yu, "ASAP: an adaptive sampling approach to data collection in sensor networks," IEEE Trans. Parallel and Distributed Systems, vol. 18, no. 12, PP. 1766-1783, 2007.

[18] C. Liu, K. Wu, and J. Pei, "An energy-efficient data collection framework for wireless sensor networks by exploiting spatiotemporal correlation," IEEE Trans. Parallel and Distributed Systems, vol. 18, no. 7, pp. 1010-1023, 2007

[19] R. Shah, S. Roy, S. Jain and W. Brunette, "Data MULEs: modeling a three-tier architecture for sparse sensor networks," Elsevier Ad Hoc Networks Journal, vol. 1, pp. 215-233, Sept. 2003.

[20] D. Jea, A.A. Somasundara and M.B. Srivastava, "Multiple controlled mobile elements (data mules) for data collection in Sensor Networks," IEEE/ACM DCOSS, June 2005.

[21] M. Ma, Y. Yang and M. Zhao, "Tour planning for mobile data gathering mechanisms in wireless sensor networks," IEEE Trans. Vehicular Technology, vol. 62, no. 4, pp. 1472-1483, May 2013.

[22] M. Zhao and Y. Yang, "Bounded relay hop mobile data gathering in wireless sensor networks," IEEE Trans. Computers, vol. 61, no. 2, pp. 265271, Feb. 2012.

[23] M. Zhao, M. Ma and Y. Yang, "Mobile data gathering with space-division multiple access in wireless sensor networks," INFOCOM 2008.

[24] M. Zhao, M. Ma and Y. Yang, "Efficient data gathering with mobile collectors and space-division multiple access technique in wireless sensor networks," IEEE Trans. Computers, vol. 60, no. 3, pp. 400-417, 2011.

[25] A. A. Somasundara, A. Ramamoorthy and M. B. Srivastava, "Mobile element scheduling for efficient data collection in wireless sensor networks with dynamic deadlines," IEEE RTSS, Dec. 2004.

[26] W. Ajib, D. Haccoun, "An overview of scheduling algorithms in MIMO-based fourth-generation wireless systems", IEEE Network, vol. 19, no. $5,2005$.

[27] S. Cui, A. J. Goldsmith and A. Bahai, "Energy-efficiency of MIMO and cooperative MIMO techniques in sensor networks," IEEE Journal on Selected Areas in Communications, vol. 22, no. 6, Aug. 2004.

[28] S. Jayaweera, "Virtual MIMO-based cooperative communication for energy constrained wireless sensor networks", IEEE Trans. on Wireless Communications, vol. 5, no. 5, 2006.

[29] S. Cui, A. J. Goldsmith and A. Bahai, "Energy-Constrained Modulation Optimization," IEEE Trans. on Wireless Communications, vol. 4, no. 5, 2005.

[30] I. Rhee, A. Warrier, J. Min, X. Song, "DRAND: Distributed Randomized TDMA Scheduling For Wireless Ad-hoc Networks", MobiHoc 06, Italy.

[31] S. C. Ergen and P. Varaiya, "TDMA scheduling algorithms for wireless sensor networks", Wireless Networks, May 2010, vol. 16, no. 4, pp 985-997.

[32] I. Rhee, A. Warrier, M. Aia and J. Min, "Z-MAC: a hybrid MAC for wireless sensor networks", ACM Sensys, 2005.

[33] D.M. Blough and P. Santi, "Investigating upper bounds on network lifetime extension for cell-based energy conservation techniques in stationary ad hoc networks," ACM MobiCom, 2002.

[34] F. Ye, G. Zhong, S. Lu and L. Zhang, "PEAS: a robust energy conserving protocol for long-lived sensor networks," IEEE ICDCS, 2003.

[35] T.H. Cormen, C. E. Leiserson, R. L. Rivest and C. Stein, Introduction to Algorithms, MIT Press, 2001.

[36] V. Tarokh, H. Jafarkhani and A.R. Calderbank, "Space-time block codes for orthogonal designs," IEEE Trans. on Info. Theory, July 1999.

[37] D. Tse and P. Viswanath, Fundamentals of Wireless Communication, Cambridge University Press, May 2005.

[38] Website: "http://www.ti.com/product/dac128s085".

[39] M. M. Solomon, “Algorithm for the vehicle routing and scheduling problems with time window constraints,"Operation Research, vol. 35, No. 2 , pp. $254-265,1987$.

\section{BIOGRAPHIES}

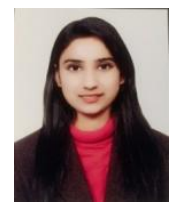

Madhu Priya, presently pursuing M.TECH in wireless communication and networks from Gautam Buddha University, Greater Noida, Uttar Pradesh. Received B.TECH in 2015 from Guru Jambheshwar University of Science and Technology, Hisar, Haryana. Area of research interest are Wireless sensor networks, Signal Processing, Computer Network, Information Security and smart antenna.

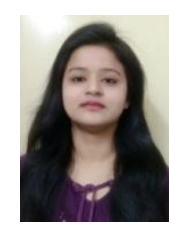

Himani Verma, presently pursuing M.TECH in wireless communication and networks from Gautam Buddha University, Greater Noida, Uttar Pradesh. Received B.TECH in 2014 from Vivekanand Institute of Technology and Science, Ghaziabad, Uttar Pradesh. Area of research interest are Wireless sensor networks, Mobile computing, Information Security, Optical system and smart antenna. 